\section{Surgically induced astigmatism after biaxial phacoemulsification compared to coaxial phacoemulsification}

R Morcillo-Laiz', MA Zato², FJ Muñoz-Negrete and F Arnalich'

Eye (2009) 23, 835-839; doi:10.1038/eye.2008.141;

published online 6 June 2008

Purpose A comparison of the topographic astigmatism generated after coaxial phacoemulsification (CP) through temporal $2.8 \mathrm{~mm}$ incision and biaxial phacoemulsification (MICS) through superioroblique trapezoidal $1.5-2 \mathrm{~mm}$ incisions. Setting Centre for Visual Sciences (Instituto de Ciencias Visuales, INCIVI), Madrid, Spain Methods This prospective randomized clinical study included 94 eyes of 64 patients; 43 eyes were operated on through $\mathrm{CP}$ and 51 through MICS. Corneal topography was measured before operation, and subsequently after 1, 3, and 6 months. Additionally, a control group (C) of 55 eyes was created (performing two topographies on them); the change in astigmatism was calculated without having performed any surgical procedure. The astigmatic change in the three groups was measured through arithmetic, polar and vector analysis (Alpins method).

Results In the vector analysis, results after the first month following surgery were: mean module of the surgically induced astigmatism (SIA) $0.49 \pm 0.38 \mathrm{D}$ in $\mathrm{CP}$ and $0.48 \pm 0.37 \mathrm{D}$ in MICS, while $0.31 \pm 0.27 \mathrm{D}$ in group C. Although no statistically significant differences were detected between the two surgical techniques, differences were noted when comparing group $C$ with each of these techniques $(P<0.05)$. The distribution of the SIA axes showed a slight tendency to be located more frequently at around $90^{\circ}$ in $\mathrm{CP}$, and at around $50^{\circ}$ in MICS.

Conclusions The mean module of SIA was similar in CP and in MICS, although the distribution of the direction of such a vector revealed minor differences.
Keywords: astigmatism; biaxial phacoemulsification; coaxial phacoemulsification; MICS

\section{Introduction}

Biaxial phacoemulsification, also known as microincisional cataract surgery (MICS), is a step forward in the evolution of cataract surgery, aiming to reduce the incision size to a minimum. This technique has several advantages, including improved fluid dynamics, associated with enhanced manoeuvrability; eg, the ability to interchange the irrigation and aspiration tips. ${ }^{1-4}$ In addition, surgery can be performed through incisions of $1.4 \mathrm{~mm},{ }^{5-6}$ or even through a $0.9 \mathrm{~mm}$ incision, ${ }^{1}$ hence inducing less postsurgery astigmatism than conventional coaxial phacoemulsification (CP). ${ }^{5-6}$

It is well recognized that in $\mathrm{CP}$, temporal clear corneal incisions (CCI) induce the least astigmatism. The ophthalmic literature estimates that the magnitude of surgically induced astigmatism (SIA) studied by vector analysis is around $0.44 \mathrm{D}$ and $0.88 \mathrm{D}$, rising as the size of the incision increases. ${ }^{7-13}$ When the axis of the SIA was studied, it was observed that temporal incisions induced 'with the rule' astigmatism. ${ }^{8,10,11}$

In this study, we aim to show whether or not MICS generates less astigmatism than CP with temporal incision. We studied astigmatism through three different methods: ${ }^{14-18}$ arithmetic, polar, and vector analysis.
'Department of Ophthalmology, Ramón y Cajal Hospital, Madrid, Spain

${ }^{2}$ Department of Ophthalmology, Centre for Visual Sciences, (Instituto de Ciencias Visuales, INCIVI), Madrid, Spain

Correspondence:

R Morcillo-Laiz,

Department of

Ophthalmology, Ramón y

Cajal Hospital,

Madrid

28023,

Spain

Tel: + 91658021 533;

Fax + 913571861

E-mail: rafaelmorcillo@

terra.es

Received: 17 October 2007 Accepted in revised form: 15 April 2008 Published online: 6 June 2008

Presented at XXI Spanish Society of Cataract and Refractive Surgery (SECOIR) Congress, Oviedo, Spain, May 2006. 


\section{Patients and methods}

Participants in this study were patients who were scheduled for elective cataract surgery. The local independent ethics committee approved the study protocol, and informed consent was obtained from each patient before being enrolled in the study. Exclusion criteria were: topographic astigmatism $>2.0 \mathrm{D}$, intraoperative use of corneal sutures, or corneal disease.

Ninety-four eyes of 64 patients (40 women and 24 men) with visually significant cataracts were enrolled in a prospective randomized controlled double-blind clinical trial to have CP with a temporal CCI or MICS; 43 eyes underwent $\mathrm{CP}$ and 51 received MICS. Neither the patient nor the examiner knew which technique had been applied.

The following examinations were performed preoperatively: measurement of best-corrected visual acuity (BCVA) using Snellen charts; slit-lamp biomicroscopy, including evaluation of nuclear lens opacity with the lens opacities classification system III (LOCS III) described by Chylack et al, ${ }^{19}$ measurement of intraocular pressure (IOP), examination of the retina, and evaluation of astigmatism by corneal topography (Zeiss-Humphrey, CA, USA).

All the operations were performed by a single surgeon (RM) under topical anaesthesia, using a two-step, self-sealing, $2.8 \mathrm{~mm}$ temporal CCI in the CP group. Two superior CCI were made at $135^{\circ}$ and $45^{\circ}$ meridians, using a trapezoidal steel keratome (Nanoedge, Geuder, Heidelberg, Germany). This forms a tunnel of $1.5 \mathrm{~mm}$ width in the endothelial area, and of $2.0 \mathrm{~mm}$ width in the epithelial surface (Figure 1). In the CP group, the intraocular lens (IOL) Y601075 (AJL, Álava, Spain) was

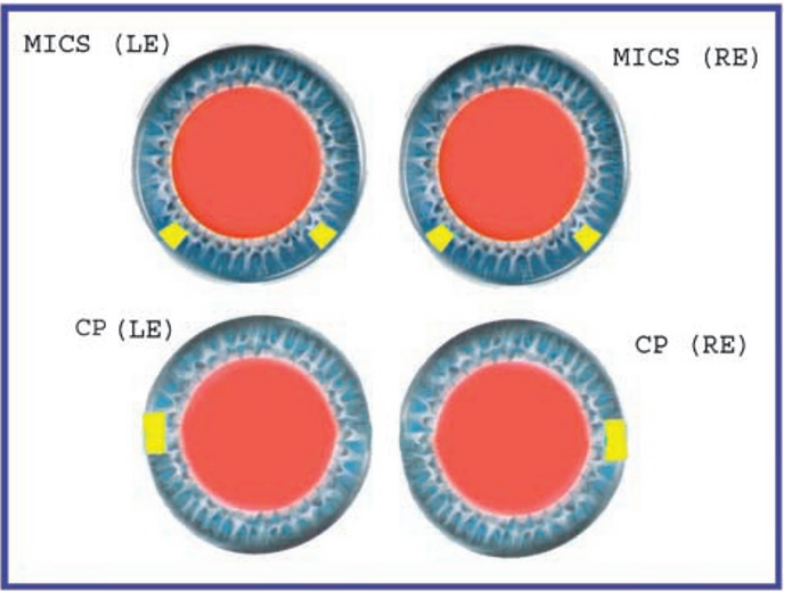

Figure 1 Location of incisions: oblique superior CCI in the MICS group, temporal CCI in the CP group; RE: right eye, LE: left eye. injected. This IOL consists of a single piece hydrophilic acrylic lens with plate haptics, a total diameter of $10.75 \mathrm{~mm}$ and $6 \mathrm{~mm}$ optic. The acrylic Acri Smart 48S IOL (Acri.Tec, Hennigsdorf, Germany) was implanted through the $135^{\circ}$ clear corneal incision in the MICS group by intraocular injection. This lens is a one-piece foldable acrylic IOL with $25 \%$ water content in a fully hydrated state and with hydrophobic surfaces. It has a $5.5 \mathrm{~mm}$ optic with a total diameter of $11 \mathrm{~mm}$. Despite its standard thickness, the lens permits an injection through a $1.5 \mathrm{~mm}$ incision because of its special design using the Acri.Smart system. This system includes an Acri.Glide cartridge and a custom-designed injector.

At 1, 3, and 6 months after surgery, BCVA was measured and astigmatism was evaluated by corneal topography. Astigmatism was studied using the following methods: ${ }^{14-17}$

(1) Arithmetic analysis, or simple subtraction, measures the amount of astigmatism before and after surgery without considering the axis.

(2) Polar analysis establishes the steepening effect occurring in the reference meridian located at $90^{\circ}$. The obtained parameter is called the vector polar netinduced value (VPNIV). A positive value indicates a 'with the rule' change in astigmatism, while a negative value illustrates an 'against the rule' change. An increase in magnitude of SIA is associated with a rise in VPNIV. This parameter accounts at the same time for the amount of change and angle of change. ${ }^{18}$

(3) Vector analysis. SIA is defined by a module and a direction.

To compare post-SIA, a control group was recruited including 55 healthy eyes. Topographic examination was performed once, and then repeated between 10-20 days afterwards, without any intervention in the interval. The same analysis previously described for the astigmatism was carried out again.

For evaluation of the astigmatism parameters, calculations were performed following the Alpins method, ${ }^{14-15}$ using the Vectrak 1.4.01 program (Victoria, Australia). All numerical and graphical evaluations were performed by means of SPSS (Version 10.0 for Windows, SPSS Inc., Chicago, IL, USA).

Comparisons of the results between the different techniques were performed by means of the non-parametric Mann-Whitney $U$-test. Comparisons of repeated measurements in continuous endpoints were evaluated by means of the Friedman test. Qualitative measurements were studied using the $\chi^{2}$ test. Results of the significance tests were summarized by means of $P$-values. $P$-values indicate local statistical significance where $P<0.05$. 
Table 1 Performed follow-up

\begin{tabular}{lccc}
\hline & 1 month & 3 months & 6 months \\
\hline CP & 43 & 40 & 38 \\
MICS & 51 & 48 & 48 \\
Total & 94 & 88 & 86 \\
\hline
\end{tabular}

$\mathrm{CP}=$ coaxial phacoemulsification; $\mathrm{MICS}=$ microincisional cataract surgery.

\section{Results}

The patients' mean age was 69.02 years \pm 8.60 in the $\mathrm{CP}$ group and $72.04 \pm 7.87$ in the MICS group. The degree of nuclear lens opacity, according to the LOCS III classification, resulted in $2.35 \pm 0.95$ and $2.43 \pm 0.90$ for the first and second groups, respectively. There were no statistical significant differences in age, sex, or nuclear lens opacity between the CP and MICS groups. The follow-up time interval range was between 1 and 6 months. At 1 month, BCVA was $0.97 \pm 0.12$ in the CP group and $0.93 \pm 0.17$ in the MICS group; no statistically significant difference was observed between both groups. Table 1 illustrates the distribution of follow-up periods.

\section{Arithmetic analysis}

Simple subtraction of astigmatism was $0.09 \pm 0.43$ in the CP group and $0.03 \pm 0.28$ in the MICS group in the first month after surgery. We found no differences between techniques when evaluating change in astigmatism using arithmetic analysis over the observational period. Mean values were all around zero with little dispersion during follow-up. The non-surgical control group showed similar results, with a change of $0.04 \pm 0.24 \mathrm{D}$, which was not statistically significant compared to both groups separately.

\section{Polar analysis}

No differences between the surgical techniques were exhibited when evaluating change in astigmatism with polar analysis. VPNIV was $0.08 \pm 0.45$ in the $\mathrm{CP}$ group and $0.07 \pm 0.42$ in the MICS group in the first month after surgery $(P=0.99)$. Polar analysis showed again values around zero, with positive values for all follow-up MICS and $\mathrm{CP}$ examinations, except at 3 months for the latter. Over the following 6 months observational period, we found that there was no difference in VPNIV, considering these techniques separately. The non-surgical control group showed similar results, a VPNIV of $0.07 \pm 0.23$, which was not statistically significant compared to both groups separately.
Table 2 SIA module

\begin{tabular}{lccc}
\hline & 1 month & 3 months & 6 months \\
\hline CP & & & \\
Mean & 0.49 & 0.44 & 0.41 \\
SD & 0.38 & 0.32 & 0.23 \\
MICS & & & \\
Mean & 0.48 & 0.5 & 0.45 \\
SD & 0.37 & 0.41 & 0.35 \\
$P$ & 0.89 & 0.77 & 0.86 \\
\hline
\end{tabular}

$\mathrm{CP}=$ coaxial phacoemulsification; $\mathrm{MICS}=$ microincisional cataract surgery; SIA $=$ surgically induced astigmatism.

\section{Vector analysis}

\section{SIA magnitude}

The SIA magnitude computed between the first month after surgery and the successive follow-up period was around $0.5 \mathrm{D}$ in both surgical groups, with little dispersion. Table 2 shows the mean SIA magnitude and the SD of both techniques during the complete follow-up period. The intergroup differences were not significant. Similarly, no significant intragroup differences were measured during the follow-up period.

In the non-surgical control group, the fictitious mean magnitude of the SIA was $0.31 \pm 0.27$ (topographic examination had been performed once, and then repeated between 10-20 days afterwards, without any intervention in the interval). This value shows statistical significance compared to both groups separately ( $P=0.015$ for $\mathrm{CP}$ and $P=0.014$ for MICS).

\section{Axis of SIA}

Axis distribution of SIA was quite homogeneous between all groups in the first month. However, as seen in the histograms (Figure 2), distribution around $90^{\circ}$ was more frequently seen in $\mathrm{CP}$, whereas distribution around $50^{\circ}$ was more frequent in MICS. By the third month, these differences became more subtle.

\section{Discussion}

The main objective of this study was to test the significant differences in surgically generated astigmatism between two phacoemulsification techniques. Modern small-incision clear corneal cataract surgery has the benefit over older techniques of causing less SIA. The use of two incisions in MICS spaced at $90^{\circ}$, superonasal and superotemporal, would cancel the coupling phenomenon associated to corneal incisions. If superior and temporal incisions were performed, the former would induce a higher degree of astigmatism than the latter. 

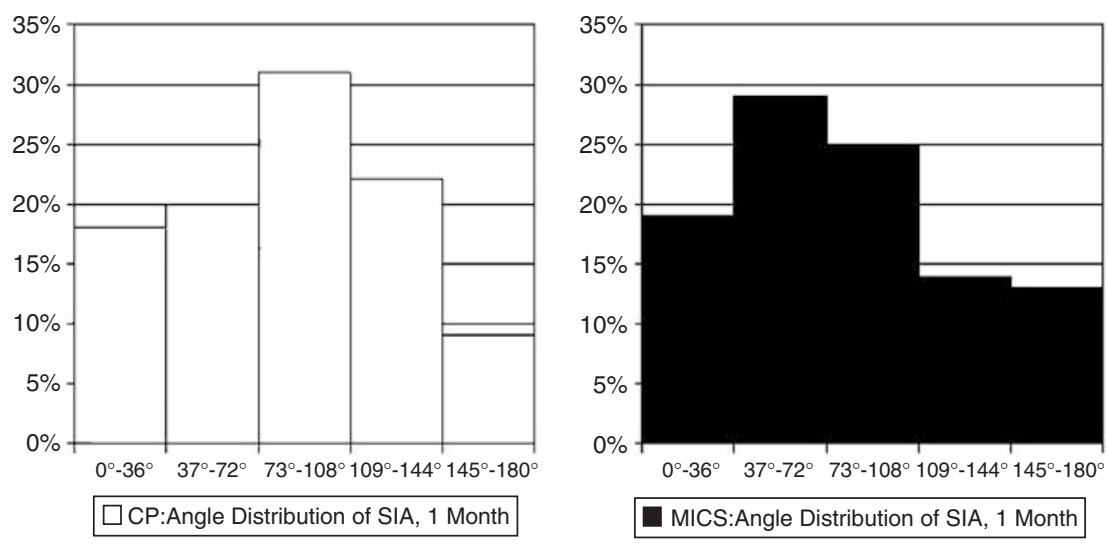

Figure 2 Axis distribution of the SIA vector in the first month.

We limited our analysis to early and late surgically induced astigmatism, up to 6 months postoperation. Astigmatism was measured using the following three methods:

Arithmetic analysis showed positive values during the whole follow-up, although the values were around zero. This showed that corneal astigmatism measured by simple subtraction was negligibly augmented. No significant differences were found between the two surgical techniques.

Polar analysis showed again values around zero, with positive values for all follow-up MICS and CP examinations, except at 3 months for the latter. The positive sign in the polar analysis shows that 'with the rule' astigmatism is more commonly induced. Once again, no significant differences were found between the two surgical techniques.

Vector analysis in the MICS and the CP groups showed mean SIA module of around 0.5D, with little dispersion. Both techniques were stable during subsequent follow-ups. No differences were observed between the two surgical techniques.

The amount of SIA for both surgical techniques has been shown not to be significantly different in our study. Alió et al, ${ }^{6}$ reported that MICS significantly lowered SIA, when compared with CP by vector analysis. However, their results are not comparable with those obtained in our work, due to the location of the incisions. Alió $e t a l^{6}$ made incisions placed at the steepest corneal meridian in both techniques; however, in this study, we systematically made temporal incisions for the CP group, which has been found to induce the least surgical astigmatism. Other authors ${ }^{20,21}$ found no difference in astigmatism with the MICS and CP groups, but results are not comparable due to the type of study design (different number, length, or localization of incisions). In common with other authors, ${ }^{7-10,22}$ we have found that the
SIA magnitude remained stable during the whole analysed period.

Axis distribution of the SIA is similar and homogeneous between techniques, although there is a trend towards $90^{\circ}$ distribution for the CP group and towards $50^{\circ}$ distribution for MICS. An axis of SIA of around $90^{\circ}$ was expected to occur in the CP group, since the incision was temporal. However, homogeneous axis distribution of SIA was expected for MICS, because we attempted to cancel out the coupling phenomenon by using the above-mentioned incision locations. An explanation for the finding of a higher frequency SIA located at $50^{\circ}$ could be that during IOL introduction in the $135^{\circ}$ meridian, the incision was forced and enlarged, which therefore produced a higher flattening effect in the $135^{\circ}$ axis, and a higher steepening effect in the $45^{\circ}$ axis. These could not be counteracted by the same amount of opposite astigmatism induced by the non-enlarged $45^{\circ}$ incision. However, we did not measure the incision at the end of the procedure.

To study whether corneal topography reproducibility or spontaneous corneal changes over time might have biased our results, we performed serial topographies on a control group.

We found that there was a change in astigmatism of $0.31 \pm 0.27$ in the case of unoperated eyes (the equivalent of SIA). Therefore, it is probable that the SIA found in our study in operated eyes might actually be smaller, since a remarkable change was found in non-surgical cases. Van den Dooren and Hennekes ${ }^{23}$ also observed a mean astigmatism of $0.35 \mathrm{D}$ in a control group of unoperated eyes. The SD of corneal power in one point is approximately $0.2 \mathrm{D} .{ }^{24}$ Changes in corneal power are also affected by diurnal and nocturnal changes as well as hormonal factors, although these are generally negligible. ${ }^{25}$

MICS potentially presents better fluid dynamics than $\mathrm{CP}$; bimanual and separated irrigation/aspiration 
functions are a safer and more comfortable procedure. Although larger studies might be necessary, we did not find significant changes in SIA when comparing MICS and $\mathrm{CP}$ performed at temporal incisions. We found the temporal approach in CP less comfortable for a righthanded surgeon, especially when operating on left eyes, as the seating position has to be changed, depending on the eye that is going to be operated on. New IOL technology that allows the easy introduction of such a lens through a $1 \mathrm{~mm}$ incision will most probably lead to a reduction in SIA in MICS.

In conclusion, the surgical techniques examined (MICS and $\mathrm{CP}$ ) generated similar levels of astigmatism in the cornea. However, given the location of the incisions, the MICS technique can be performed by the surgeon more comfortably while positioned in the head area of the patient than when using the lateral position expected in the CP through a temporal corneal incision.

\section{References}

1 Agarwal A, Agarwal A, Agarwal S, Narang P, Narang S. Phakonit: Phacoemulsification through a $0.9 \mathrm{~mm}$ corneal incision. J Cataract Refract Surg 2001; 27: 1548-1552.

2 Soscia W, Howard JG, Olson RJ. Microfacoemulsification with WhiteStar. A wound-temperature study. J Cataract Refract Surg 2002; 28: 1044-1046.

3 Donnenfeld ED, Olson RJ, Solomon R, Finger PT, Biser SA, Perry HD et al. Efficacy and wound-temperature gradient of White Star phacoemulsification through a $1.2 \mathrm{~mm}$ incision. J Cataract Refract Surg 2003; 29: 1097-1100.

4 Fine IH, Hoffman RS, Packer M. Optimizing refractive lens exchange with bimanual microincision phacoemulsification. J Cataract Refract Surg 2004; 30: 550-554.

5 Tsuneoka H, Shiba T, Takahashi Y. Ultrasonic phacoemulsification using a $1.4 \mathrm{~mm}$ incision: clinical results. J Cataract Refract Surg 2002; 28: 81-86.

6 Alió J, Rodríguez-Prats JL, Galal A, Ramzy M. Outcomes of microincision cataract surgery versus coaxial phacoemulsifiction. Ophthalmology 2005; 112: 1997-2003.

7 Masket S, Tennen DG. Astigmatic stabilization of $3.0 \mathrm{~mm}$ temporal clear corneal cataract incisions. J Cataract Refract Surg 1996; 22: 1451-1455.

8 Rainer G, Menapace R, Vass C, Annen D, Findl O, Schmetterer K. Corneal shape changes after temporal and superolateral $3.0 \mathrm{~mm}$ clear corneal incisions. J Cataract Refract Surg 1999; 25: 1121-1126.
9 Barequet IS, Yu E, Vitale S, Cassard S, Azar DT, Stark WJ. Astigmatism outcomes of horizontal temporal versus nasal clear corneal incision cataract surgery. J Cataract Refract Surg 2004; 30: 418-423.

10 Demirok A, Çinal A, Yilmaz ÖF. Effect of superior and temporal clear corneal incisions on astigmatism after sutureless phacoemulsification. J Cataract Refract Surg 1998; 24: 515-518.

11 Lyhne N, Krogsager J, Corydon L, Kjeldgaard M. One-year follow-up of astigmatism after $4.0 \mathrm{~mm}$ temporal clear corneal and superior scleral incisions. J Cataract Refract Surg 2000; 26: 83-87.

12 Müller-Jensen K, Buchholz A, Barlinn B. Stability of astigmatism over 3 years after corneal stretch incision. J Cataract Refract Surg 1998; 14: 455-459.

13 Müller-Jensen K, Barlinn B. Long-term astigmatic changes after clear corneal cataract surgery. J Cataract Refract Surg 1997; 23: 354-357.

14 Alpins NA. A new method of analyzing vectors for changes in astigmatism. J Cataract Refract Surg 1993; 19: 524-533.

15 Alpins N. Astigmatism analysis by the Alpins method. J Cataract Refract Surg 2001; 27: 31-49.

16 Koch DD. How should we analyze astigmatic data? Editorial J Cataract Refract Surg 2001; 27: 1-3.

17 Alpins NA, Goggin M. Refractions and reflections. Surv Ophthalmol 2004; 49: 109-122.

18 Naeser K. Assessment of surgically induced astigmatism; call for an international standard. (letter) J Cataract Refract Surg 1997; 23: 1278-1280.

19 Chylack L, Wolfe J, Singer D. Lens opacities classification system III. Arch Ophtalmol 1993; 111: 831-833.

20 Kurz S, Krummenauer F, Gabriel P, Pfeiffer N, Dick HB. Biaxial microincision versus coaxial small-incision clear cornea cataract surgery. Ophthalmology 2006; 113: 1818-1826.

21 Cavallini GM, Campi L, Masini C, Pelloni S, Pupino A. Bimanual microphacoemulsification versus coaxial miniphacoemulsification: Prospective study. J Cataract Refract Surg 2007; 33: 387-392.

22 Nielsen PJ. Prospective evaluation of surgically induced astigmatism and astigmatic keratotomy effects of various self-sealing small incisions. J Cataract Refract Surg 1995; 21: $43-48$.

23 Van den Dooren K, Hennekes R. Vector analysis of surgically induced astigmatism in small corneal and scleral cataract incisions. Bull Soc Belge Ophthalmol 1996; 262: 167-171.

24 Hannush SB, Crawford SL, Waring III GO, Gemmill MC, Lynn MJ, Nizam A. Accuracy and precision of keratometry, photokeratoscopy, and corneal modeling on calibrated steel balls. Arch Ophthalmol 1989; 107: 1235-1239.

25 Corbett MC, Rosen ES, O'Brart DPS. Normal topography. In: Corneal Topography. London: BMJ Books, 1999, pp 63-79. 